\title{
RADIATIONLESS TRANSITIONS
}

\author{
JOSHUA JORTNER \\ Department of Chemistry, Tel-Aviv University, Tel-Aviv, Israel
}

\begin{abstract}
This review will be concerned with some questions which arise whenever one thinks of analyzing experimental radiative decay times and optical line shape data in polyatomic molecules, and how these quantities are related to fundamental electronic relaxation processes in a large molecule.
\end{abstract}

\section{INTRODUCTION}

Radiationless processes in excited electronic states of large molecules can be classified as follows:

(a) Radiationless decomposition ${ }^{1}$ :

(a1) Molecular predissociation.

(a2) Molecular autoionization.

(b) Intramolecular relaxation ${ }^{2}$ :

(b1) Electronic relaxation processes which involve internal conversion and intersystem crossing.

(b2) Unimolecular photochemical rearrangement reactions in excited electronic states of large molecules such as cis-trans isomerization or electrocyclic reactions.

The present review will be concerned mainly with electronic relaxation processes. From the historical point of view it has been known since 1888 that many organic molecules in a dense medium exhibit a strong afterglow (or rather, phosphorescence) when excited by ultraviolet light, this emission invariably being at lower frequencies than the fluorescence (if any) of the compound ${ }^{3}$. These observations were followed in 1933 by the phenomenological description of the three level system by Jablanski ${ }^{4}$. The modern focus on the importance and generality of intramolecular relaxation processes was emphasized by the work of Lewis and coworkers and by Kasha ${ }^{5}$. During the last twenty years extensive studies were performed which elucidated some important features of electronic relaxation processes of polyatomic molecules imbedded in a dense medium (e.g. solutions, rigid glasses, mixed crystals) ${ }^{2}$. These studies resulted in several generalizations:

(a) The Kasha rules ${ }^{2}$.

(b) 'Shortening' of the experimental radiative lifetimes of large molecules, accompanied by a reduction of the emission quantum yield ${ }^{2}$.

(c) The Robinson-Frosch ${ }^{6}$ energy gap law.

(d) The deuterium isotope ${ }^{6,7}$.

In view of the bulk of experimental data concerning radiationless transitions in a dense medium it is not surprising that early theories by Gouterman ${ }^{8}$ and by Robinson and Frosch ${ }^{6}$ considered the molecule-medium coupling 
as an essential ingredient which will provide a pathway for electronic relaxation. An impetus for a drastic revision of theoretical ideas concerning this problem was provided (as usual in theoretical chemistry!) by recent experimental data ${ }^{9-13}$ which demonstrate conclusively that radiationless transitions occur in an 'isolated' low density gas phase molecule. About three years ago theoretical studies by Henry and Kasha ${ }^{2 d}$, Robinson ${ }^{14}$ and Bixon and Jortner ${ }^{15}$ provided a firm basis for the idea that a radiationless transition in a large molecule involves an intramolecular relaxation process.

I would like to discuss some aspects of recent work on the interpretation of optical line shape data and radiative decay times in large molecules, and how this information relates to intramolecular electronic relaxation processes in large molecules.

\section{COMMENTS ON COMPOUND STATES}

How does a relaxation process take place in a microscopic system? Three major points have to be amplified in this context:

(a) The description of the decaying state.

(b) The preparation of the metastable nonstationary state.

(c) The relation between the decay time and the optical line shapes.

Suppose that the system under consideration is described by the Hamiltonian

$$
H=H_{0}+V
$$

where $H_{0}$ is a 'convenient' zero order Hamiltonian (e.g. independent particles Hamiltonian in the case of autoionization ${ }^{16}$, of the Born-Oppenheimer Hamiltonian in the case of predissociation) ${ }^{17-19}$, while $V$ is a perturbation term which includes whatever we have left out of $H_{0}$. The zero order eigenstates of $H_{0}$ are now partitioned into two sets: a (dynamic) sparse subset which is characterized by a small number of discrete levels $\phi_{1}, \phi_{2}$ and a dissipative part $\phi_{E}$ which is characterized by a continuous spectrum. As the zero order states of the two subsystems are degenerate, extensive 'configuration interaction' is induced by the (small) interaction term $V$ which couples the dynamic and the dissipative part. An atom or molecule in a stationary state cannot make transitions to the other states which are induced by the 'small terms in the molecular Hamiltonian' (i.e. $V$ in equation (II.1).) Obviously all time dependent transitions between stationary states are radiative in nature. However this conclusion does not apply when the molecular system is prepared by some experiments in a nonstationary state of the system's Hamiltonian. To obtain the physical information concerning the relaxation process it will be convenient to proceed as follows ${ }^{16,19}$ :

(a) The molecular eigenstates $\psi_{E}$ of the system are constructed (e.g. the eigenstates of $H$ in the absence of the radiation field) as a time independent superposition of the zero order states. For convenience we shall consider only a single state $\phi_{1}$ in the dynamic subset, so that

$$
\psi_{E}=a(E) \phi_{1}+\int b_{E^{\prime}}(E) \phi_{E^{\prime}} \rho\left(E^{\prime}\right) \mathrm{d} E^{\prime}
$$

where $\rho(E)$ is the density of states in the zero order continuum, and $a(E)$ and 


\section{RADIATIONLESS TRANSITIONS}

$\left\{b_{E^{\prime}}(E)\right\}$ correspond to expansion coefficients. Note that the eigenstates $\phi_{E}$ form a continuous spectrum.

(b) The resonance width $\Gamma(E)$ is given for a single resonance by the Fermi Golden Rule:

$$
\Gamma(E)=2 \pi\left|\left\langle\phi_{1}|V| \psi_{E}\right\rangle\right|^{2} \rho(E)
$$

For the case of a dissipative continuum one expects that it is a slowly varying function of the energy in the vicinity of the $E=E_{1}$, which is the energy of the zero order state $\phi_{1}$.

(c) The Breit Wigner Formula. The amplitude square of the zero order state $\phi_{1}$ is given by the distribution:

$$
|a(E)|^{2}=(\Gamma / 2) /\left[\left(E-E_{1}-\gamma_{1}\right)^{2}+(\Gamma / 2)^{2}\right]
$$

where $\gamma_{1}$ is a level shift. Thus the effect of configuration interaction is to 'dilute' the discrete state $\phi_{1}$ through a manifold of stationary states. The profile of the distribution is a Lorentzian characterized by the width:

$$
\Delta=\Gamma
$$

(d) The transition matrix element of the transition operator $\hat{T}$ for optical (or other type) excitation from the ground state $\phi_{0}$ is determined by:

$$
\left\langle\phi_{0}|\hat{T}| \psi_{E}\right\rangle=a(E)\left\langle\phi_{0}|\hat{T}| \phi_{1}\right\rangle+\int b_{E^{\prime}}(E)\left\langle\phi_{0}|\hat{T}| \phi_{E^{\prime}}\right\rangle \rho\left(E^{\prime}\right) \mathrm{d} E^{\prime}
$$

(e) The line shape $A(E)$ for optical excitation will be determined by the square of the transition matrix element:

$$
A(E) \propto\left|\left\langle\phi_{0}|\hat{T}| \psi_{E}\right\rangle\right|^{2}
$$

(f) Lorentzian line shapes. The absorption profile will be Lorentzian only, provided that the continuum does not carry oscillator strength, i.e. $\left\langle\phi_{0}|\hat{T}| \phi_{E^{\prime}}\right\rangle=0$ for all $\phi_{E^{\prime}}$. Then

$$
A(E) \propto|a(E)|^{2}=\left(1 / 1+\varepsilon^{2}\right)
$$

where the reduced energy parameter $\varepsilon$ is

$$
\varepsilon=E-E_{1}-\gamma_{1} /(\Gamma / 2)
$$

(g) Fano type line shapes. In the general case, when the continuum does carry oscillator strength from the ground state, interference effects in absorption will be observed arising from the contributions of $\left\langle\phi_{0}|\hat{T}| \phi_{1}\right\rangle$ and $\left\langle\phi_{0}|\hat{T}| \phi_{E^{\prime}}\right\rangle$ which will interfere with opposite phases on the two sides of the resonance. The line shape function will then be

$$
A(E) \propto \frac{(q+\varepsilon)^{2}}{\left(1+\varepsilon^{2}\right)}
$$

where the line profile index $q$ is determined by the ratio transition moments for the discrete state and for the continuum.

$$
q \approx \frac{\left\langle\phi_{0}|\hat{T}| \phi_{1}\right\rangle}{\left\langle\phi_{0}|\bar{T}| \phi_{E}\right\rangle\left\langle\phi_{E}|V| \phi_{1}\right\rangle \rho(E)}
$$


Note that equation (II.10) is reduced to the Lorentzian form (II.8) provided that $q \rightarrow \infty$. This situation will be realized when:

(1) the coupling between the zero order states is negligible.

(2) when the oscillator strength of the background absorption is negligibly small, relative to the intensity carried by $\phi_{1}$.

(h) A compound state of the system will be described as a time dependent superposition of time independent zero order states. The choice of the basis set is merely a matter of convenience. One possible choice of the basis set involves the stationary states of $H$ or, alternatively, the basis set of $H_{0}$ may be used. Let $p(E)$ correspond to the excitation amplitude of $\psi_{E}$, then two alternative forms for the time dependent excited state can be immediately written:

$$
\Psi(t)=\int p(E) \psi_{E} \exp (-i E t / \hbar) \rho(E) \mathrm{d} E \equiv A(t) \phi_{1}+\int B_{E}(t) \phi_{E} \rho(E) \mathrm{d} E
$$

It should be noted that the compound state involves an admixture of zero order discrete and continuum states.

(i) The time evolution of the amplitude of the discrete state can then be given

$$
P(t)=\left|\left\langle\phi_{1} \mid \Psi(t)\right\rangle\right|^{2}=\left|\int \rho(E) a(E) \exp (-i E t / \hbar) \rho(E) \mathrm{d} E\right|^{2} \equiv|A(t)|^{2}
$$

Thus the decay law is determined by an energy distribution function. It should be noted that, unlike the line shapes which are definitely experimental observables, the quantity $\boldsymbol{P}(t)$ may not always be amenable to experimental observation.

(i) Decay of state 'prepared' initially in $\phi_{1}$.

The $|A(0)|^{2}=1$ or $p(E)=a(E)$; provided that $\Gamma(E)$ is a slowly varying function of the energy on exponential decay law results:

$$
P(t) \propto \exp (-\Gamma t / \hbar)
$$

The half lifetime is just $\hbar \Gamma^{-1}$ and the simple decay law is related to the width of the amplitude distribution $|a(E)|^{2}$. This is again a 'theoretical exercise' which will not always be realized in a real life experiment.

The foregoing arguments are general, leading to the conclusion that there is a set of features common to all compound states of a wide class of systems. The shapes of resonances encountered in nuclear, atomic, molecular and solid-state physics are nearly the same and the decay rates of many different kinds of metastable states have the same functional form.

For radiationless decomposition processes the dissipative channel is well defined (e.g. a dissociative continuum for the case of predissociation and an ionization continuum for autoionization). The details of the relaxation process (e.g. decay times and line shapes) will be determined by the coupling matrix elements, the transition moments and the line profile index. At first sight it may appear that there is an apparent basic difference between radiationless decomposition and intramolecular relaxation as in the latter case

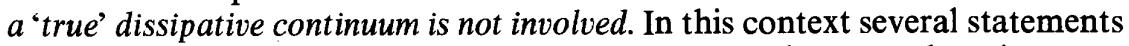
have been made concerning non-radiative intramolecular relaxation processes. It was stated by Herzberg ${ }^{20}$ in 1966: 'The mechanism of (internal) conversion is not well understood as yet but is presumably connected with 
strong perturbations between the two states involved.' At about the same time Kistiakowsky and Parmenter ${ }^{13}$ stated that their experimental observation of a radiationless transition in the isolated benzene molecule 'may be incompatible with the laws of quantum mechanics'. The questions that come up in relation to intramolecular relaxation processes in large molecule can be summarized as follows:

(a) What is the nature of the intramolecular coupling?

(b) Do radiationless transitions take place in an isolated large molecule?

(c) What is the nature of the intramolecular dissipative channel?

(d) What are the criteria for irreversibility of an intramolecular relaxation process?

(e) What are the implications of intramolecular coupling and a background quasicontinuum of states concerning intensity distribution in optical absorption?

(f) Under what conditions can the intramolecular decay be considered as a simple rate process with the rate constant being given by Fermi's 'Golden Rule'?

(g) What are the consequences of the coupling between radiative and non-radiative decay processes in a large molecule?

\section{INTRAMOLECULAR COUPLING}

The nature of the intramolecular coupling responsible for radiationless transitions was elucidated many years ago by Franck and Sponer ${ }^{21}$ and by $\mathrm{Kubo}^{22}$ who pointed out that the nuclear kinetic energy operator provides the major interaction term which is responsible for the occurrence of radiationless processes in large molecules. Naturally, other intramolecular interaction terms may modify the mixing. Thus, for example, spin-orbit interactions have to be included in the case of mixing of quasidegenerate vibronic components which correspond to two electronic states of different multiplicity.

The electronic states of a molecule are conventionally classified within the framework of the Born-Oppenheimer approximation, into separate electronic and nuclear motions. Let us focus our attention on the conventional Born-Oppenheimer (BO) adiabatic approximation for the two level system.

The higher excited electronic state, $s$, is characterized by the zero-order BO levels $\phi_{s \alpha}(r, Q)$ which are coarsely spaced, each of which is coupled to the dense quasicontinuum of vibronic levels $\phi_{l \beta}(r, Q)$. These functions are usually approximated in the form:

$$
\begin{aligned}
& \phi_{s \alpha}(\boldsymbol{r}, \boldsymbol{Q})=\Theta_{s}(\boldsymbol{r}, \boldsymbol{Q}) \chi_{s \alpha}(\boldsymbol{Q}) \\
& \phi_{l \beta}(\boldsymbol{r}, \boldsymbol{Q})=\Theta_{l}(\boldsymbol{r}, \boldsymbol{Q}) \chi_{l \beta}(\boldsymbol{Q})
\end{aligned}
$$

where $r$ represents the electronic coordinates, while $Q \equiv Q_{1}, Q_{2}, \ldots Q_{k}$ ... (III.1) correspond to the nuclear coordinates. $\Theta$ and $\chi$ represent electronic and vibrational wavefunctions. The potential surfaces in the two electronic states will be denoted by $E_{s}(Q)$ and $E_{1}(Q)$ while the energies of the vibronic components will be represented by $E_{s a}$ and $E_{1 \beta}$, respectively.

As is well known, the $\mathrm{BO}$ representation is diagonal within a single 
electronic manifold while (hopefully small) matrix elements connect different electronic states. These off diagonal matrix elements are given in the general form:

$$
\begin{aligned}
v_{s \alpha, l \beta}=\frac{1}{2} \sum_{k} & \int \boldsymbol{Q}_{\chi_{s \alpha}}(Q)\left\langle\boldsymbol{\Theta}_{s}(\boldsymbol{q}, \boldsymbol{Q})\left|\frac{\partial^{2}}{\partial Q_{k}^{2}}\right| \Theta_{l}(\boldsymbol{q}, \boldsymbol{Q})\right\rangle_{l \beta}(\boldsymbol{Q}) \\
& -\sum_{\boldsymbol{k}} \int \mathrm{d} \boldsymbol{Q} \chi_{s \alpha}(\boldsymbol{Q})\left\langle\boldsymbol{\Theta}_{s}(\boldsymbol{q}, \boldsymbol{Q})\left|\frac{\partial}{\partial Q_{k}}\right| \Theta_{l}(\boldsymbol{q}, \boldsymbol{Q}) \frac{\partial}{\partial Q_{k}} \chi_{l \beta}(\boldsymbol{Q})\right\rangle
\end{aligned}
$$

The electronic matrix elements appearing in (III.2) can be expressed in the exact form:

$$
\begin{aligned}
J_{s l}^{k} & =\Theta_{s}(\boldsymbol{q}, \boldsymbol{Q})\left|\frac{\partial}{\partial Q_{k}}\right| \Theta_{l}(\boldsymbol{q}, \boldsymbol{Q}) \\
& =\frac{\left\langle\Theta_{s}(\boldsymbol{q}, \boldsymbol{Q})\left|\partial U(\boldsymbol{q}, \boldsymbol{Q}) / \partial Q_{k}\right| \Theta_{l}(\boldsymbol{q}, \boldsymbol{Q})\right\rangle}{E_{s}(\boldsymbol{Q})-E_{l}(\boldsymbol{Q})}
\end{aligned}
$$

where $U(\boldsymbol{q}, \boldsymbol{Q})$ corresponds to the molecular potential energy term.

The breakdown of the BO approximation will be encountered under the following circumstances:

(a) Strong interaction between degenerate or quasidegenerate electronic origins. This situation corresponds to the Jahn-Teller and Renner coupling in molecules.

(b) Intersection of potential surfaces: the electronic matrix element (III.3) is a rapidly varying function of the nuclear coordinates, whereupon near the intersection (where $E_{s}(Q)-E_{l}(Q)=0$ ) a new representation of the vibronic wave functions has to be found in a manner analogous to the treatment of the Jahn-Teller problem. Such situations which involve a large configurational change between two electronic states will be encountered in the field of organic photochemistry.

(c) Case of near degeneracy. Now we encounter small configurational change between two electronic states. The electronic integral $J_{s l}^{k}$ is a slowly varying function of $Q$ and we expect that $J_{s l}^{k} \alpha \Delta E^{-1}$, where $\Delta E=E_{s 0}-E_{10}$ corresponding to the electronic energy gap between the origins of the two electronic states. $\Delta E$ is appreciable and the $v_{s a, l \beta}$ terms are small not only in view of the energy denominator but rather as they involve extremely small $^{6,23-26}$ Franck-Condon vibrational overlap terms. However the smallness of the $v$ terms does not insure the validity of the BO approximation. The adiabatic approximation is expected to hold only provided the energy difference between the zero order vibronic states is large relative to the coupling matrix element (III.2) so that

$$
\left|E_{s \alpha}-E_{l \beta}\right| \gg\left|v_{s \alpha, l \beta}\right|
$$

When a situation of near degeneracy is encountered we expect the BO approximation to break down even provided that the $v$ terms are small.

Let us now consider the behaviour of the excited electronic levels of a complex molecule. The zero order vibronic level $\phi_{s}$ of a higher excited state 


\section{RADIATIONLESS TRANSITIONS}

is quasidegenerate with a manifold $\left\{\phi_{l}\right\}$ of vibronic levels which correspond the lower excited electronic states and to the ground state. The density of vibronic states of these lower configurations is determined by two factors:

(a) The number of vibrational degrees of freedom.

(b) The energy gap between the zeroth vibronic levels of the two electronic states.

The density of vibronic states at energy $\Delta E$ above the origin of an electronic state can be approximated by the semiclassical expression in the harmonic approximation $^{27}$ :

$$
\rho=\frac{\Delta E^{n-1}}{(n-1) !} \prod_{i=1}^{n}\left(h v_{i}\right)^{-1}\left(1+\frac{h}{2} \sum_{i} v_{i} / \Delta E\right)^{-1}
$$

where $n$ is the number of the vibrational degrees of freedom, characterized by the frequencies $\gamma_{i}$. To obtain some feeling for the order of magnitude of the density of these background states we have displayed in Table 1 estimates

Table 1. Density of vibrational states in a series of hypothetical polyatomic molecules where all $v_{i}=1000 \mathrm{~cm}^{-1}$ and $\Delta E=1 \mathrm{eV}$.

\begin{tabular}{cc}
$\begin{array}{l}\text { No. of } \\
\text { atoms }\end{array}$ & $\begin{array}{l}\rho \\
\mathrm{cm}\end{array}$ \\
\hline 3 & 0.06 \\
4 & 4 \\
5 & 50 \\
6 & 400 \\
10 & $4 \times 10^{5}$ \\
\hline
\end{tabular}

of the vibrational density of levels in a hypothetical polyatomic molecules, characterized by an energy gap of $1 \mathrm{eV}$, while in Table 2 we have assembled

Table 2. Densities of vibronic states in some aromatic hydrocarbons.

\begin{tabular}{lcccc}
\hline System & lower state & upper state & $\begin{array}{c}\Delta E \\
\mathrm{~cm}^{-1}\end{array}$ & \multicolumn{1}{c}{$\begin{array}{c}\rho \\
\mathrm{cm}\end{array}$} \\
\hline Anthracene & & ${ }^{1} B_{2 u}$ & 12000 & $5 \times 10^{10}$ \\
Napthalene & ${ }^{3} B_{2 u}$ & ${ }^{1} B_{2 u}$ & 3400 & $2 \times 10^{3}$ \\
Napthalene & ${ }^{1} B_{3 u}$ & ${ }^{3} B_{2 u}$ & 20000 & $8 \times 10^{15}$ \\
Azulene & ${ }^{1} A_{1}$ & ${ }^{1} B_{1}$ & 14000 & $10^{11}$ \\
Benzene & ${ }^{3} B_{1 u}$ & ${ }^{1} B_{2 u}$ & 8400 & $8 \times 10^{4}$ \\
\hline
\end{tabular}

some data for real physical systems. Obviously in view of the overwhelmingly large densities of vibronic states encountered in large molecules we expect that even small coupling matrix elements (equation III.2) will lead to appreciable level mixing. Under these common circumstances, when a discrete zero order level is quasidegenerate with a background manifold of vibronic states, the Born-Oppenheimer separability conditions break down. 
Thus in general the excited electronic states of large molecules cannot be considered as 'pure' BO states.

One has to distinguish very carefully between the consequences of intramolecular coupling and intramolecular relaxation. In particular, it should be borne in mind that coupling can be exhibited while relaxation does not occur. We shall, therefore, attack the problem in two steps which will be analogous to the general problem of relaxation already considered.

(a) The molecular eigenstates of the system will be constructed ${ }^{28-30,2 a, 15}$.

(b) The conditions for irreversible decay will be then established ${ }^{30-32,15}$.

\section{MOLECULAR EIGENSTATES}

A proper representation of the molecular eigenstates $\psi_{n}$, can be obtained from a superposition of zero order Born-Oppenheimer states.

$$
\psi_{n}=a_{s}^{n} \phi_{s}+\sum_{l} b_{l}^{n} \phi_{l}
$$

The Hamiltonian is given by

$$
H_{e l}=H_{B 0}+H_{V}
$$

where $H_{B 0}$ corresponds to the Born-Oppenheimer Hamiltonian while $H_{V}$ contains the nuclear kinetic energy, spin-orbit coupling etc. $\phi_{s}$ and $\left\{\phi_{l}\right\}$ are eigenstates of $H_{B O}$. Notice how the near degeneracy of levels in a large molecule resembles the situation encountered in the treatment of the pseudo Jahn-Teller effect. However, in the present case the coupling between many quasidegenerate zero order states must be considered rather than that between only a few states. The configuration interaction scheme ${ }^{15,28}$ employed herein is similar to the treatments employed many years ago by Rice ${ }^{17}$ in the study of predissociation, and by Fano ${ }^{16}$ in the study of autoionization. However, it should be stressed that unlike the cases of autoionization and predissociation, in the present case the dense manifold of states is discrete.

The model I would like to discuss is grossly oversimplified but transparent. First we assume that the levels in the $\left\{\phi_{1}\right\}$ manifold are equally spaced with spacing $\rho^{-1}$. This is not too bad. However the second assumption is rather serious. We shall assume that all the coupling terms are equal and set $v_{s l}=\left\langle\phi_{s}\left|H_{e l}\right| \phi_{1}\right\rangle=v$. This immediately leads to a simple eigenvalue problem:

$$
\left(\begin{array}{llc}
E_{s}-E & v & v \ldots \\
v & E_{1}-E & 0 \ldots \\
v & 0 & E_{2}-E \ldots \\
\vdots & \vdots & \vdots
\end{array}\right)\left(\begin{array}{c}
a_{s} \\
b_{1} \\
b_{2} \\
\vdots
\end{array}\right)=0
$$

The solutions for the energies $E_{n}$ of the molecular eigenstates (III.1) can be obtained from the equation:

$$
E_{s}-E_{n}-\pi v^{2} \rho \operatorname{Cotan}\left[\pi \rho\left(\dot{E}_{s}-E_{n}\right)\right]=0
$$

which can be solved numerically. The expansion coefficients representing 


\section{RADIATIONLESS TRANSITIONS}

the weights of the zero order states $\phi_{s}$ in the molecular eigenstate $\psi_{n}$ are given by:

$$
\left|a_{s}^{n}\right|^{2}=\frac{v^{2}}{\left(E_{n}-E_{s}\right)^{2}+v^{2}+\left(\pi v^{2} \rho\right)^{2}}
$$

Two points have to be made at this stage:

(a) The choice of the BO basis set to describe the molecular eigenstates is arbitrary but extremely useful. In principle all the eigenstates of $H_{0}$ have to be included in (IV.1). In the BO representation the admixture of higher excited states (whose electronic origin is located above $\phi_{s}$ ) will be very small. On the other hand if we had chosen a poor zero order representation which involves electronic wavefunctions at a fixed nuclear configuration (the 'crude adiabatic approximation' $)^{34}$ the admixture of these higher states which are not quasidegenerate with $\phi_{s}$ would have become important.

(b) The zero-order $\mathrm{BO}$ state $\phi_{s}$ plays a special role as this state carries oscillator strength from the ground state while the manifold $\left\{\phi_{l}\right\}$ is devoid of oscillator strength. Thus the intensity distribution in absorption will be determined by $\left|a_{s}^{n}\right|^{2}$.

The following comments should be made at this point concerning the square of the expansion coefficient (IV.5):

(a) The distribution is Lorentzian.

(b) The width $\Delta$ of the distribution is given by the dominant term in the denominator which is either $v^{2}$ or $\left(\pi v^{2} \rho\right)^{2}$.

(c) The condition for strong interstate coupling is

$$
v \rho \geqslant 1
$$

This condition implies, of course, that $\Delta$ is determined by $v^{2} \rho$ rather than by $v$ itself. Equation (IV.6) will be valid provided that one of the following situations is realized:

(c1) Strong interaction with a sparse manifold or, alternatively,

(c2) Relatively weak coupling with a dense manifold. Conditions (c1) or (c2) imply that the width of the distribution will be determined by the simple relation

$$
\Delta=\pi v^{2} \rho
$$

(d) The criterion (IV.7) for strong mixing is just equivalent to the breakdown of the BO approximation (equation III.4).

When the strong electronic coupling condition applies, the intensity (in absorption) of the zero order states $\phi_{s}$ is distributed among a manifold of molecular eigenstates. Two cases have to be considered :

(1) Coarsely spaced molecular eigenstates. The levels are well separated relative to their radiative and inhomogenous widths ${ }^{2 e}$. The individual transition moments will be given by :

$$
\left|\left\langle\phi_{0}|\boldsymbol{\mu}| \psi_{M}\right\rangle\right|^{2}=\left|a_{s}^{n}\right|^{2}\left|\left\langle\phi_{0}|\boldsymbol{\mu}| \phi_{S}\right\rangle\right|^{2}
$$

Thus the intensity is distributed over a coarsely spaced well resolved manifold of states.

(2) The statistical limit. The molecular eigenstates are densely spaced relative to their rediative widths and equation (IV.6) is valid. Now, we cannot 
expect to resolve individual levels in the optical spectrum. The following implications are evident :

(2a) The line shape in absorption is:

$$
A(E) \propto \rho\left|a_{s}^{n}\right|^{2}=\frac{v^{2} \rho}{\left(E-E_{s}\right)^{2}+\left(\pi v^{2} \rho\right)^{2}}
$$

(2b) The absorption line shape is Lorentzian, the half line width being given by (VI.7).

(2c) The Lorentzian line shape is due to the fact that the background continuum does not carry oscillator strength.

(2d). The situation in this case is completely analogous to the Lorentzian distribution of amplitudes and the line shape obtained for a single resonance which results from the interaction with a 'real' continuum (see section II). Thus the dense quasicontinuum acts as an effective continuum.

This situation will be referred to as The Statistical Limit.

To conclude this discussion it is interesting to point out that the description, presented herein, of the strong interstate coupling in molecules bears a close resemblance to the problem of intermediate structure in nuclear reactions ${ }^{35}$. In the latter case compound states of the nucleus are constructed as a superposition of a single excitation and more complex excitations in a manner completely analogous to equation (IV.1). The single excitation which can be reached via the incident channel is referred to as a 'doorway state' and is formally analogous to the BO state $\phi_{s}$ in the molecular case.

\section{DIFFUSENESS AND INTERFERENCE EFFECTS IN THE ELECTRONIC SPECTRA OF LARGE MOLECULES}

We shall now consider the implications of the effects of intramolecular interstate coupling in molecules for the understanding of the intensity distribution in absorption.

(a) Strongly coupled sparse manifold. Singlet excited states of small molecules such as $\mathrm{SO}_{2}, \mathrm{NO}_{2}$ or $\mathrm{CS}_{2}$ are quasidegenerate with a relatively low density of vibronic states belonging to the lower triplet state and the ground state. However, because of favourable Franck-Condon vibrational overlap factors (due to changes in the molecular geometry in the excited states) the vibronic coupling terms are quite large. Thus, we expect that $v \rho>1$. The occurrence of vibronic coupling in moderately small molecules implies the redistribution of the intensity of the zero order component $\phi_{s}$, and this redistribution induces the appearance of many new lines (corresponding to all the molecular eigenstates $\psi_{n}$ ) into the spectrum. A situation of this type probably explains the high resolution spectrum of $\mathrm{NO}_{2}$, where a large number of irregularly spaced lines is observed. The general conclusions cited concur with those of Douglas ${ }^{36}$.

(b) The statistical limit: The breakdown of the $\mathrm{BO}$ approximation in the statistical limit results in line broadening which arises from a differential distribution of intensity among a large number of closely spaced molecular eigenstates. This 'diffuseness' of the spectral lines occurs as an intramolecular phenomenon.

What are the experimental implications of this result for spectroscopic studies of large molecules? 


\section{RADIATIONLESS TRANSITIONS}

(a) Intravalence excitations. Intramolecular coupling with a dense vibronic manifold leads to broadening of higher excited states arising from intravalence excitations in large molecules. To assess the role of intramolecular coupling on the line broadening 'trivial' broadening effects have to be eliminated. In the elegant work of Ross et al..$^{37}$ the following 'irrelevant' broadening mechanisms in the gas phase spectra of large molecules were considered :

(i) Doppler width

(ii) Rotational broadening

(iii) Spectral congestion

(iv) Photo dissociation

(v) Non-radiative decompositions (autoionization and predissociation).

In solid-state spectra of molecules trapped in low temperature matrices effects (i), (ii) and (iii) are missing; however the following additional sources of broadening have to be taken into account:

(vi) Phonon broadening, which can be eliminated by utilization of Spolski matrices for the observation of zero phonon lines.

(vii) Vibrational relaxation of higher vibronic components.

(viii) Site splittings.

Ross et $a l^{23-25,38}$ have systematically demonstrated that no trivial mechanism can explain the diffuseness of higher intravalence excitations in the gas phase.

(b) Extravalence excitations. The situation with respect to line broadening is radically different when extravalence excitations, such as transitions to molecular Rydberg states in large molecules are considered. In the gas phase, these absorption lines corresponding to the Rydberg levels are quite sharp ${ }^{39}$. Thus, for example, the line widths of the $3 R$ Rydberg states of benzene are of the order of a few $\mathrm{cm}^{-1}$, i.e. about one or two orders of magnitude lower than the line widths of the $\pi \rightarrow \pi^{* 1} A_{1 g} \rightarrow{ }^{1} E_{1 u}$ transition. The situation is reminiscent of relatively weak vibronic coupling in these Rydberg states. This observation can be easily rationalized by noting that the vibronic coupling terms involve one electron operator of the form $\sum_{k} \partial V / \partial Q_{k}$ where $V$ is the molecular coulomb potential energy while $\left\{Q_{k}\right\}$ correspond to the nuclear coordinates (see Section IV). Hence the coupling between the large radius Rydberg orbital and the ground state orbital via the $\partial V / \partial Q_{k}$ terms is expected to be relatively weak.

(c) Interference between resonances. Even when the background quasicontinuum does not carry intensity interesting effects are expected to be encountered when the widths of several Lorentzians (e.g. several vibrational components in a given electronic state) exceed their spacings. Under these circumstances, we cannot limit ourselves to a single resonance as interference effects between resonances are expected to be encountered ${ }^{31}$. No definite experimental evidence for this effect in molecular spectra is at present available.

(d) Intermediate structure: Electronic states of large molecules which are characterized by a small electronic energy gap reveal some interesting structure in the optical absorption. Thus the second singlet excited state of the naphthalene molecule which is separated by about $3000 \mathrm{~cm}^{-1}$ from the 
first singlet exhibits some relatively sharp lines superimposed on a diffuse background ${ }^{40}$. This fine-structure is sensitive to the nature of the host crystal (which affects the energy gap) and to the isotopic composition of the molecule. Now, it is obvious that in real life not all the states in the $\left[\phi_{r}\right\}$ manifold couple to the $\phi_{s}$ with the same strength. In the statistical limit this problem is of minor importance; however in the present intermediate case these strongly coupled levels will borrow most of the oscillator strength and will be resolved in the spectrum.

(e) Interference with background absorption. Up to this point we have considered only an isolated resonance. In this case, the 'background' states $\left\{\phi_{1}\right\}$ do not carry out oscillator strength so that no Fano-type interference effects are expected to be revealed in the optical spectrum. An interesting relevant situation is encountered when Rydberg levels overlap an inhomogeneously broadened $\pi \rightarrow \pi^{*}$ transition. Such a situation prevails for the $2 R$ Rydberg state of benzene which in the gas phase is quasidegenerate with the ${ }^{1} A_{1 g} \rightarrow{ }^{1} E_{1 u} \pi \rightarrow \pi^{*}$ transition ${ }^{41}$. In the case of the naphthalene molecule, the $n=5$ to $n=13$ Rydberg levels overlap a medium intensity $(f \sim 0.1)$ transition located near $62000 \mathrm{~cm}^{-1}$. The gas phase optical spectrum of napthalene, as reported recently by Angus, Christ and Morris ${ }^{41}$, reveals several sharp antiresonances. Morris and Jortner ${ }^{42}$ discussed the nature of the interference effects which give rise to this unique behaviour in the optical spectrum of an isolated large molecule ${ }^{42}$ between resonance and potential scattering.

\section{INTRAMOLECULAR NON-RADIATIVE DECAY}

We now study some of the consequences of statistical mixing, and consider the time development of coherently excited states. The molecule in the ground state is subjected to a radiative perturbation, which in the dipole approximation is

$$
H^{\prime}(t)=\boldsymbol{\mu} \cdot \varepsilon \delta(t)
$$

where $\varepsilon$ is the electric field acting on the molecule. For simplicity we have used a delta function excitation. The excited state at time $t=0$ can be described in terms of a superposition of molecular eigenstates:

$$
\Psi(t=0)=\sum_{n} \mu_{0 n} \psi_{n}
$$

where $\mu_{0 n}$ is the transition dipole moment to the molecular eigenstate $\psi_{n}$, which can be displayed in the form

$$
\mu_{0 n}=a_{s}^{n} \mu_{s 0}
$$

The wave function at time $t$ is given by

$$
\Psi(t)=\sum_{n} \mu_{0 n} \psi_{n} \exp \left(-i E_{n} t / \hbar\right)
$$

Consider now the time development of the amplitude of the zero order state $\phi_{s}$ in the excited state which is given by

$$
P(t)=\left|\left\langle\phi_{s} \mid \Psi(t)\right\rangle\right|^{2}=\frac{\mu_{0 s}^{2}}{\hbar^{2}}|s(t)|^{2}
$$


In the kernel $S(t)$ is :

$$
S(t)=\sum_{n}\left|a_{s}^{n}\right|^{2} \exp \left(-i E_{n} t / \hbar\right)
$$

so that the relaxation rate will be determined by the Fourier transform of the line shape function $\left|a_{s}^{n}\right|^{2}$. Under the limiting conditions

$$
\begin{aligned}
& v \rho \gg 1 \\
& t \ll \hbar \rho
\end{aligned}
$$

The relaxation process is exponential

$$
P(t) \propto \exp \left(-t / \tau_{n r}\right)
$$

where the non-radiative decay time is given by the Fermi Golden Rule:

$$
\tau_{n r}=\hbar / 2 \pi v^{2} \rho
$$

Up to this point we have considered the relaxation process within the framework of a simple model system. A more general treatment can be easily performed which, as in the case of the absorption coefficient (section V), will lead to a more general criterion for the validity of the statistical limit. Consider again the alternative representation of $\Psi(t)$ in terms of the BO basis set. Making use of the orthonormality properties of the expansion coefficients $a_{s}^{n}$ and $b_{i}^{n}$ in equation (IV.1), the initial state (VI.2) can then be represented in the form:

$$
\Psi(t=0)=\mu_{0 s} \phi_{s}
$$

The time evolution of the excited state can now be displayed as the time dependent superposition

$$
\Psi(t)=A(t) \phi_{s}+\sum_{l} B_{l}(t) \phi_{l}
$$

Making use of conventional time dependent perturbation theory results in the equation of motion for the amplitude $A(t)$ :

$$
\begin{array}{r}
\hbar^{2} \dot{A}(t)=-\int_{0}^{t} \mathrm{~d} t^{\prime} A\left(t^{\prime}\right) \sum_{l}\left|v_{s l}\right|^{2} \exp \left[\frac{i\left(E_{l}-E_{s}\right)\left(t-t^{\prime}\right)}{\hbar}\right] \\
=-2 \pi \iint \mathrm{d} E \mathrm{~d} t^{\prime} A\left(t^{\prime}\right) \sum_{l}\left|v_{s l}\right|^{2} \exp \left[\frac{i\left(E-E_{s}\right)\left(t-t^{\prime}\right)}{\hbar}\right] \dot{\partial}\left(E-E_{l}\right) \\
=-\iint \mathrm{d} E \mathrm{~d} t^{\prime} A\left(t^{\prime}\right) \Delta(E) \exp \left[\frac{i\left(E-E_{s}\right)\left(t-t^{\prime}\right)}{\hbar}\right]
\end{array}
$$

where

$$
\Delta(E)=2 \pi \sum_{l}\left|v_{s l}\right|^{2} \delta\left(E-E_{l}\right)
$$

In the statistical limit $\Delta(E)$ is the slowly varying function of the energy $\mathrm{y}^{33,43}$ and thus we set it to be a constant $\Delta=\Delta(E)$. The expression for $\Delta(E)$ in the 
statistical limit is just the half line width (IV.7). One now immediately obtains the exponential decay law:

$$
|A(t)|^{2}=|A(0)|^{2} \exp \left(-t / \tau_{n r}\right)
$$

\section{IRREVERSIBLE INTRAMOLECULAR DECAY IN THE STATISTICAL LIMIT}

When the background density of vibronic states in a large molecule is extremely high this manifold is expected to act as an effective continuum with respect to line broadening and to intramolecular relaxation. The general criteria obtained for the statistical limit can be summarized as follows :

$$
\begin{gathered}
v \rho \gg 1 \\
t \ll \hbar \rho \\
\Delta(E)=2 \pi \sum_{l}\left|v_{s l}\right|{ }^{2} \delta\left(E-E_{l}\right)
\end{gathered}
$$

is smooth.

It should be borne in mind that while conditions (VII.1) and (VII.2) were obtained for a simple model system, equation (VII.3) is general and model independent. The simple model calculations provide us with physical insight concerning the general features of the non-radiative decay process which can be summarized as follows:

(a) Equation (VII.1) provides a necessary condition for line broadening and for the occurrence of intramolecular non-radiative decay: however, this energetic condition is by no means sufficient.

(b) The relation (VII.2) establishes the time scale for the occurrence of the non-radiative decay. In fact $t_{R}=\hbar \rho$ corresponds to the recurrence time for the decay of the zero order level $\phi_{s}$ into the quasicontinuum. For times longer than $t_{R}$, the amplitude of $\phi_{s}$ in $\Psi(t)$ will increase towards its initial value. However, for large molecules these recurrence times considerably exceed the time scale of any experiment.

(c) The definition of the recurrence time introduces the notion of irreversibility of the intramolecular radiationless process. This recurrence time introduces a Poincare cycle for the irreversible process. An intramolecular radiationless process in the limit of a sufficiently large density of vibronic levels corresponds to an irreversible process on a time scale which is shorter than $t_{R}$.

(d) Electronic relaxation in large molecules (see Table 3) obeys the restrictions (VII.1) and (VII.2). Thus these can be considered as legitimate intramolecular relaxation phenomena.

Obviously the simple relations (VII.1) and (VII.2) are gross oversimplifications based on a 'coarse graining' procedure. Let us consider now a real physical system where the necessary and sufficient condition for irreversible non-radiative decay is given by the 'smoothness' of $\Delta(E)$ (equation VII.3). This restriction is more general and enables us to ascertain the salient features of the intramolecular decay in a large molecule. We note that a 'hidden assumption' involved in the simple model calculations implies that the zero states $\left\{\phi_{l}\right\}$ have zero widths. If these levels are characterized by 


\section{RADIATIONLESS TRANSITIONS}

Table 3. Parameters descriptive of radiationless transitions in large molecules.

\begin{tabular}{lccccc}
\hline System & $\begin{array}{c}\tau_{\mathrm{nr}} \\
\mathrm{sec}\end{array}$ & $\begin{array}{c}v \\
\mathrm{~cm}^{-1}\end{array}$ & $\begin{array}{c}\rho \\
\mathrm{cm}\end{array}$ & $v \rho$ & $\begin{array}{c}h \rho \\
\mathrm{sec}\end{array}$ \\
\hline $\begin{array}{l}\text { Anthracene } \\
{ }^{1} B_{2 u}-{ }^{3} B_{2 u} \\
E=12000 \mathrm{~cm}^{-1}\end{array}$ & $5 \times 10^{-9}$ & $6 \times 10^{-7}$ & $5 \times 10^{10}$ & $3 \times 10^{10}$ & 0.25 \\
$\begin{array}{l}\text { Napthalene } \\
{ }^{3} B_{2 u}-{ }^{1} A_{1 g} \\
E=20000 \mathrm{~cm}^{-1}\end{array}$ & 2 & $10^{-14}$ & $8 \times 10^{15}$ & 80 & $4 \times 10^{4}$ \\
$\begin{array}{l}\text { Azulene } \\
{ }^{1} B_{1}-{ }^{1} A_{1}\end{array}$ & $6 \times 10^{-11}$ & $2 \times 10^{-5}$ & $10^{11}$ & $2 \times 10^{6}$ & 0.5 \\
$\begin{array}{l}E=14000 \mathrm{~cm}^{-1} \\
\text { Benzene }\end{array}$ & $10^{-6}$ & $1.5 \times 10^{-5}$ & $8 \times 10^{4}$ & 1.5 & $4 \times 10^{-7}$ \\
${ }^{1} B_{2 u}-{ }^{3} B_{1 u}$ & & & & &
\end{tabular}

finite widths $\left\{\boldsymbol{\Gamma}_{l}\right\}$ then the resonance width should be altered by replacing each delta function in the sum (VII.3) by a Lorentzian ${ }^{33,43}$ :

$$
\Delta(E)=\sum_{l} \frac{\left(\boldsymbol{\Gamma}_{l} / 2\right)\left|v_{s l}\right|^{2}}{\left(E-E_{l}\right)^{2}+\left(\boldsymbol{\Gamma}_{l} / 2\right)^{2}}=I_{m} \sum_{l} \frac{\left|v_{s l}\right|^{2}}{\left(E-E_{l}\right)+i \Gamma_{l} / 2}
$$

Obviously when $\boldsymbol{\Gamma}_{l} \rightarrow 0^{+}$we regenerate equation (VII.3). In order to consider an upper limit $t_{m}$ for the decay process, Freed ${ }^{43}$ adds an imaginary part $i \hbar / t_{m}$ to the energy $E$ so that

$$
E \rightarrow E+\frac{\hbar}{t_{m}}
$$

Such a trick is common in scattering theory and amounts to describing the decay process in terms of a (complex) Green's function $G\left(E+i \hbar / t_{m}\right)$. Usually one sets $\hbar / t_{m} \rightarrow 0^{+}$; however as pointed out by Freed ${ }^{43}$, this is not really necessary, as the inclusion of the imaginary factor introduces a term of the form $\exp \left(-t / t_{m}\right)$ in the decay process and thus erases all the behaviour of the system for long times, e.g. $t \gg t_{m}$.

The general form of the resonance width is then

$$
\Delta(E)=\sum_{l} \frac{\left|v_{s l}\right|^{2}\left(\frac{\Gamma_{l}}{2}+\frac{\hbar}{t_{m}}\right)}{\left(E-E_{l}\right)^{2}+\left(\frac{\Gamma_{l}}{2}+\frac{\hbar}{t_{m}}\right)^{2}}
$$

This result exhibits a superposition of generalized Lorentzians, each characterized by the strength $\left|v_{s l}\right|^{2}$ and by the width

$$
\frac{\Gamma_{l}}{2}+\frac{\hbar}{t_{m}}
$$

A general condition for the smoothness of $\Delta(E)$ is that the widths of successive Lorentzians considerably exceed their spacings. Stated mathematically 


$$
\begin{gathered}
\text { JOSHUA JORTNER } \\
\frac{\Gamma_{l}}{2}+\hbar / t_{m} \gg E_{l+1}-E_{l} \sim \hbar / \rho
\end{gathered}
$$

Hence the general condition for irreversibility will be displayed in the form:

$$
\rho^{-1} \ll \hbar / t_{m}+\Gamma_{l} / 2
$$

The following cases should now be considered:

(a) Intersystem crossing in the lowest triplet in an isolated molecule. In this case the line widths $\Gamma_{l}$ are negligibly small, as the levels $\left\{\phi_{l}\right\}$ do not carry oscillator strength to the ground state or to any of its vibronic components. Setting $\Gamma_{l} \rightarrow 0^{+}$one immediately obtains the simple relation (VII.2).

(b) Internal conversion in an isolated molecule. Now the dense manifold $\left\{\phi_{l}\right\}$ is connected by nonvanishing radiative coupling terms to high vibronic levels of the ground state. This is, of course, the reason for the observation of fluorescence radiation from the second (and any higher) singlet to high vibrational levels of the ground state. Hence $\Gamma_{l}>0$. Two pertinent cases have to be considered:

(a2) A large energy gap between two excited states of the same multiplicity. Thus for example the first $\left({ }^{1} B_{2 u}\right)$ and the second $\left({ }^{1} B_{3 u}^{+}\right)$singlet excited states of anthracene are separated by $\Delta E=15000 \mathrm{~cm}^{-1}$. In this case we expect that

$$
\Gamma_{l} \gg \rho^{-1}
$$

and the function $\Delta(E)$ is smooth on any time scale as we can set $t_{m} \rightarrow \infty$.

(b2) A small energy gap between two states of the same multiplicity. A good example in this category involves the ${ }^{1} B_{2 u}$ and the ${ }^{1} B_{3 u}$ excited states of the naphthalene molecule where the energy gap is $\Delta E=3400 \mathrm{~cm}^{-1}$. In this case $\rho^{-1} \sim 10^{-3} \mathrm{~cm}^{-1}$. Now $\rho^{-1} \gg \Gamma_{l}$ and again the condition $t_{m} \ll \hbar \rho$ has to be applied for the decay of the ${ }^{1} B_{2 u}$ state.

(c) Internal conversion and intersystem crossing in a condensed medium. Getting away from the isolated molecule and considering for a moment the medium effects at low temperatures, we note that now $\Gamma_{l}$ has a substantial contribution $\left(10^{-2}-10^{-1} \mathrm{~cm}^{-1}\right)$ due to vibrational relaxation. Hence the condition $\Gamma_{l} \gg \rho^{-1}$ is usually satisfied. We can neglect $t_{m}^{-1}$ with respect to $\Gamma_{l}$ and the decay process is irreversible for long times. Obviously, states which correspond to intermediate cases for the molecule in the low pressure gas phase will reveal irreversible decay when the molecule is embedded in a medium.

To summarize this discussion, the following relations are of interest:

(a) For the case of intersystem crossing

$$
\tau_{\text {rad }}(l) \gg \hbar \rho \gg t_{m} \gg \tau_{\text {rad }}(s)
$$

(b1) For internal conversion (large energy gap):

$$
\infty \leftarrow t_{m} \gg \hbar \rho \gg \tau_{\text {rad }}(l) \sim \tau_{\text {rad }}(s) .
$$

(b2) For internal conversion (small energy gap):

$$
\tau_{\text {rad }}(s) \sim \tau_{\text {rad }}(l) \gg \hbar \rho \gg t_{m}
$$

(c) For a molecule in a dense medium:

$$
\infty \leftarrow t_{m} \gg \hbar \rho \gg \tau_{\text {rad }}(s) \geqslant \tau_{v, r}(l)
$$




\section{RADIATIONLESS TRANSITIONS}

where $\tau_{\text {rud }}$ and $\tau_{v, r}$ correspond to lifetimes due to radiative decay and to vibrational relaxation, respectively.

\section{THE COUPLING BETWEEN RADIATIVE AND NON RADIATIVE PROCESSES IN LARGE MOLECULES}

A large bulk of physical information now available concerning intramolecular coupling and electronic relaxation in polyatomic molecules comes from lifetimes of molecular luminescence. Clearly, a complete theoretical description of the radiationless transition process should emerge from the description of the radiative decay. It should be recalled that we are now considering a phenomenon associated with the decay of a manifold of a large number of closely spaced levels ${ }^{44-53}$ (e.g. the molecular eigenstates).

We shall now consider a simplified version of the theory of the radiative decay of polyatomic molecules ${ }^{29-33}$. One pedantic comment should be made at this point concerning the molecular eigenstates representation. When radiative decay processes are considered the molecular eigenstates are no longer proper eigenstates of the Hamiltonian

$$
H=H_{e l}+H_{n}+H_{i n+}
$$

As before, the molecular Hamiltonian, $H_{e l}$, consists of the BO term $H_{B O}$ and an intramolecular perturbation $H_{v}$ (vibronic, spin-orbit, etc.). $H_{r}$ is the Hamiltonian corresponding to the free radiation field while $H_{\text {int }}$ is the radiation-matter interaction term. The time evolution of a nonstationary state of the system can be described either in terms of the eigenstates of $H_{B O}$ (the BO basis set) or of $H_{e l}$ (the molecular eigenstates basis). Obviously, the choice of the basis set is merely a matter of convenience and thus does not affect any observable quantities. The questions that have to be answered by a complete study of the radiative decay of a polyatomic molecule are as follows:

(a) Are simple kinetic schemes, as applied for years by the experimentalist, adequate?

(b) When will interference effects be observed in the radiative decay?

(c) How can details of the decay process (e.g. quantum yields and experimental radiative lifetimes) be elucidated?

In order to handle the radiative decay of a large molecule, consider the initial excited state at time $t=0$ which is a nonstationary state of $H$, and no photons are present. The initial excited molecular state $\Psi_{m}(0)$ can always be expressed as a superposition of either the molecular eigenstates $\left\{\psi_{m}\right\}$ or the BO states $\phi_{s},\left\{\phi_{l}\right\}$. The initial state of the system is :

$$
\begin{aligned}
\Psi(0)=\Psi_{m}(0)|v a c\rangle=\sum_{n} a_{n}(0)\left|\psi_{n} ; v a c\right\rangle= & b_{s}(0)\left|\phi_{s} ; v a c\right\rangle \\
& +\sum_{l \neq s} b_{l}(0)\left|\phi_{l} ; v a c\right\rangle
\end{aligned}
$$

where $|v a c\rangle$ is the zero photon state. In many cases of physical interest the initial excited state of the system can be visualized as being prepared by a coherent excitation, by a short light pulse or by a chaotic broad band source whereupon:

$$
\begin{aligned}
& a_{n}(0)=\left\langle\phi_{s} \mid \psi_{n}\right\rangle \\
& b_{l}(0)=\delta_{l s}
\end{aligned}
$$


Obviously, the completeness of the molecular eigenstates basis and the fact that $\phi_{s}$ is the only state which carries oscillator strength immediately imply that in this case:

$$
\Psi(0)=\sum_{n}\left\langle\phi_{s} \mid \psi_{n}\right\rangle\left|\psi_{n} ; v a c\right\rangle \equiv\left|\phi_{s} ; v a c\right\rangle
$$

We now proceed to provide a simple description of the decay process. A more elaborate treatment was recently provided by Freed and Jortner. The final states of the system consist of one photon ground state $\left.\phi_{k, e}=\phi_{0} ; \boldsymbol{k}, \boldsymbol{e}\right)$ where $\phi_{0}$ is the ground electronic state while $\boldsymbol{k}$ and $\boldsymbol{e}$ correspond to the wave vector and the polarization vector of the emitted photon. The time dependent state of the system is given by:

$$
\begin{array}{r}
\Psi(t)=\sum_{n} a_{n}(t)\left|\psi_{n} ; v a c\right\rangle+\sum_{\boldsymbol{k}} \sum_{\boldsymbol{e}} c_{\boldsymbol{k} e}(t)\left|\phi_{0} ; \boldsymbol{k}, \boldsymbol{e}\right\rangle \equiv b_{s}(t)\left|\phi_{s} ; v a c\right\rangle \\
+\sum_{l \neq s} b_{l}(t)\left|\phi_{l} ; v a c\right\rangle+\sum_{\boldsymbol{k}} \sum_{\boldsymbol{e}} d_{\boldsymbol{k} \boldsymbol{e}}(t) \mid \phi_{0} ; \boldsymbol{k}, \boldsymbol{e}
\end{array}
$$

with the initial conditions given by equation (VIII.3) and $c_{k, e}(0)=d_{k},(0)=0$ for all $\boldsymbol{k}$ and $e$. The probability $A_{s}(t)$ for the decay of the system is given by:

$$
A_{\mathrm{s}}(t)=|\langle\Psi(0) \mid \Psi(t)\rangle|^{2}
$$

Making use of the initial conditions (VIII.3) we get for the decay rate of the excited state, which corresponds to the total number of photons emitted per unit time:

$$
\dot{Q}(t)=\frac{\Gamma_{s}}{\hbar}\left|A_{s}(t)\right|^{2}=\frac{\Gamma_{s}}{\hbar}\left|\sum_{n} a_{n}(t) a_{n}(0)\right|^{2} \equiv \frac{\Gamma_{s}}{\hbar}\left|b_{s}(t)\right|^{2}
$$

where $\Gamma_{s}$ is the radiative width of the zero order state $\phi_{s}$ equation (VIII.7) reveals the following features of the decay process:

(a) When the BO basis set is employed we have to focus our attention on the decay channels of the zero order state $\phi_{s}$.

(b) When the molecular eigenstates basis is used the decay rate contains a contribution from interference effects between closely spaced levels.

Equation (VIII.5) provides us with a proper description of the time dependent compound state of the system which is presented as a superposition of time independent zero order states. In order to elucidate the features of the decay process we have to establish the equations of motion for the coefficients $\left\{a_{n}(t)\right\}$ or $\left\{b_{s}(t), b_{l}(t)\right\}$. This can be accomplished by the following methods:

(a) A self-consistent extension of the Wigner-Weisskopf method ${ }^{54}$ to account for the decay of a large number of levels ${ }^{30}$.

(b) The 'unitary relations' method employed in the field of elementary particles physics ${ }^{56,57}$ which is based on general conservation rules ${ }^{30}$.

(c) The Fano configuration interaction method ${ }^{16}$ whereupon the radiation field provides a dissipative continuum ${ }^{30,31}$.

(d) The Green's function method adopted to the decay of a large number of metastable levels ${ }^{33}$. 


\section{RADIATIONLESS TRANSITIONS}

All these methods lead to the following result: let the time dependent compound state be given in the general form

$$
\Psi(t)=\sum_{j} \alpha_{j}(t)\left|\chi_{j} ; v a c\right\rangle+\sum_{\boldsymbol{k}} \sum_{\boldsymbol{e}} \beta_{\boldsymbol{k}, \boldsymbol{e}}(t)\left|\phi_{\boldsymbol{k}, \boldsymbol{e}}\right\rangle
$$

where the set $\chi_{j}$ is any general complete set (molecular eigenstates, BO basis or other). If we define the row vector

the equation of motion is ${ }^{30,55}$

$$
\alpha(t)=\left(\begin{array}{c}
\alpha_{1}(t) \\
\alpha_{2}(t) \\
\vdots
\end{array}\right)
$$

$$
\begin{aligned}
& i \frac{\mathrm{d}}{\mathrm{d} t} \boldsymbol{\alpha}(t)=\boldsymbol{H}_{e f f} \boldsymbol{\alpha}(t) \\
& \boldsymbol{H}_{\text {eff }}=\boldsymbol{H}_{e l}-\frac{1}{2} \boldsymbol{\Gamma}
\end{aligned}
$$

where $\Gamma$ corresponds to the damping matrix. In discussing phenomena of radiative decay it is customary to introduce the radiative lifetime of states. The damping matrix $\boldsymbol{\Gamma}$ is defined for some (arbitrary) set of zero photon excited states in the form:

$$
\Gamma_{j j^{\prime}}=(2 \pi / \hbar) \sum_{\boldsymbol{e}} \int \mathrm{d} \Omega_{\boldsymbol{k}}\left\langle\chi_{j} ; v a c\left|H_{i n t}\right| \phi_{\boldsymbol{k}, \boldsymbol{e}}\right\rangle\left\langle\phi_{\boldsymbol{k}, \boldsymbol{e}}\left|H_{i n t}\right| \chi_{j^{\prime}} ; v a c\right\rangle \rho_{k}
$$

where $\sum_{e} \int \mathrm{d} \Omega_{\mathrm{k}}$ corresponds to the integration overall propagation direction in the $k$ space and summation over all polarization directions of the emitted photon. $\rho_{k}$ is the density of photon states. Thus equations (VIII.10) and (VIII.12) provide us with the general decay law for a manifold of closely spaced levels. The following comments are now in order:

(a) The damping matrix provides a generalization of the Fermi 'Golden Rule' transition rates.

(b) The damping matrix $\boldsymbol{\Gamma}$ is in general non-diagonal.

(c) The matrix $\boldsymbol{H}_{\text {eff }}$ which determines the decay is non-hermitian (or rather antihermitian). This observation can be rationalized by noting that equation (VIII.10) factors out only a finite number of (zero photon) states of the system instead of considering the infinite number of states which characterize the Hamiltonian (VIII.1).

(d) When a non-diagonal representation of $\boldsymbol{H}_{\text {eff }}$ is employed (which is usually the case) the states $\left|\chi_{j} ; v a c\right\rangle$ do not decay independently, e.g. they cannot be characterized by simple exponential decays. This is the case provided that the off diagonal terms of the damping matrix are large, so that

$$
\Gamma_{j j^{\prime}} \gtrsim \mid E_{j}-E_{j^{\prime}}-\frac{i}{2}\left(\Gamma_{j j}-\Gamma_{j^{\prime} j^{\prime}} \mid\right.
$$

This effect is known in level crossing, where the decaying states are indistinguishable (e.g. characterized by the same symmetry).

(e). In principle, one can find a set of zero photon states characterized by 
exponential decay, provided that $\boldsymbol{H}_{e f f}$ is diagonalized by a complex orthogonal matrix $S$, so that

$$
\boldsymbol{S H} \boldsymbol{H}_{\text {eff }} \boldsymbol{S}^{-1}=\boldsymbol{A}
$$

The real and imaginary parts of the diagonal matrix $\boldsymbol{A}$ provide us with the energies and lifetimes respectively of the states for which $\boldsymbol{H}_{\text {eff }}$ is diagonal.

(f) In the BO basis $\left\{\phi_{s}, \phi_{l}\right\}$ the effective Hamiltonian is:

$$
\left(\begin{array}{cccc}
\left(E_{s}-\frac{i \Gamma_{s}}{2}\right) & v_{s 1} & v_{s 2} & \cdots \\
v_{s 1} & E_{1} & 0 & \cdots \\
v_{s 2} & 0 & E_{2} & \cdots \\
\vdots & \vdots & \vdots &
\end{array}\right)
$$

so that $\boldsymbol{H}_{e l}$ is off diagonal while the damping matrix is diagonal.

(g) For the molecular eigenstate $\left\{\psi_{n}\right\}$ basis the effective Hamiltonian is given in the form:

$$
\left(\begin{array}{ccc}
E_{1}-\frac{i \Gamma_{11}}{2} & -\frac{i \Gamma_{12}}{2} & \cdots \\
-\frac{i \Gamma_{21}}{2} & E_{2}-\frac{i \Gamma_{22}}{2} & \cdots \\
\vdots & \vdots &
\end{array}\right)
$$

Now $\boldsymbol{H}_{e}$ is diagonalized; however, we pay the price by having the damping matrix in a non-diagonal representation.

This general formalism can be immediately applied for the following cases:

Table 4. Long radiative lifetimes of small molecules.

\begin{tabular}{clcc}
\hline Molecule & Transition & $\begin{array}{c}\tau \text { (expt.) } \\
\text { sec }\end{array}$ & $\begin{array}{c}\tau(\text { integrated } f \text { ) } \\
\text { sec }\end{array}$ \\
\hline $\mathrm{NO}_{2}$ & ${ }^{1} B_{2}-{ }^{1} A_{1}$ & $44 \times 10^{-6}$ & $0.3 \times 10^{-6}$ \\
$\mathrm{SO}_{2}$ & $4300 \AA$ & $42 \times 10^{-6}$ & $0.2 \times 10^{-6}$ \\
& $3000 \AA$ & & $3 \times 10^{-6}$ \\
$\mathrm{CS}_{2}$ & ${ }^{1} B_{1}-{ }^{1} A_{1}$ & $15 \times 10^{-6}$ & \\
& $3200 \AA$ & & \\
& ${ }^{1} \Sigma-{ }^{1} \Sigma$ & & \\
& ${ }^{1} \pi-{ }^{1} \Sigma$ & & \\
\hline
\end{tabular}

(i) Long radiative lifetimes of triatomic molecules (see Table 4). Under these circumstances the levels in the $\left\{\phi_{l}\right\}$ manifold are coarsely spaced, considerably exceeding the radiative widths of the molecular eigenstates. Application of the molecular eigenstates basis implies that for the off diagonal elements of the damping matrix

$$
\Gamma_{n n^{\prime}} \ll\left|E_{n}-E_{n^{\prime}}-\frac{i}{2}\left(\Gamma_{n n}-\Gamma_{n^{\prime} n^{\prime}}\right)\right|
$$




\section{RADIATIONLESS TRANSITIONS}

so that these off diagonal terms are negligible. Thus in this limit we get:

$$
\left(\boldsymbol{H}_{e f f}\right)_{n n^{\prime}}=\left(E_{n}-\frac{i}{2} \Gamma_{n}\right) \delta_{n n^{\prime}}
$$

where $\Gamma_{n}$ is the radiative width of the molecular eigenstate $\psi_{n}$ so that :

$$
\Gamma_{n} \equiv \Gamma_{n n}=\Gamma_{s}\left|\left\langle\psi_{n} \mid \phi_{s}\right\rangle\right|^{2}=\Gamma_{n}\left|a_{s}^{n}\right|^{2}
$$

where the coefficient $a_{s}^{n}$ is given by equation (IV.1).

The decay law is given by a sum of exponentials

$$
\dot{Q}(t)=\sum_{n}\left|a_{n}(0)\right|^{2} \exp (-\Gamma n t / \hbar)
$$

Since $\left|a_{s}^{n}\right|^{2}<1$ we have from equation (VIII.19)

$$
\Gamma_{n}<\Gamma_{s} \text { for all } n
$$

We thus have the explanation for the anomalously long radiative lifetimes of small molecules ${ }^{30}$. The occurence of vibronic coupling in triatomic molecules implies the redistribution of intensity of the zero order component $\phi_{s}$ and the 'dilution' of the decay times of the molecular eigenstates each of which now decays independently.

(ii) Short radiative lifetimes of large molecules.

In the statistical limit the decay law is

$$
\dot{Q}(t)=\left(\Gamma_{s} / \hbar\right) \exp \left(-\left\{\Gamma_{s}+\Delta / \hbar\right\} t\right)
$$

Thus the radiative decay in the statistical limit is exponential and the experimental radiative decay time consists of independent contributions from non-radiative and radiative components.

The quantum yield determined on a time scale appreciably shorter than the recurrence time is given in the form:

$$
Y=\Gamma_{\mathrm{s}} / \Gamma_{s}+\Delta
$$

This does not imply that the large molecule acts as a photon trap, but rather that only a fraction $Y$ of photons will be emitted on the time scale $t \ll \hbar \rho$, or stated more generally, on the time scale $t \ll t_{m}$ (see section VII).

To conclude this discussion of the statistical limit we should notice that two legitimate complementary descriptions of the decay of an excited state of a large molecule can be given:

(1) Interference effects between a large number of closely spaced zero order levels (e.g. the molecular eigenstates) give rise to the shortening of the radiative lifetime.

(2) The excited state corresponds to a resonance which is coupled to two different continua. Just as the photon continuum allows for irreversible radiative decay, the $\left\{\phi_{l}\right\}$ manifold acts as a second dissipative channel.

To conclude this discussion we shall briefly consider the general features of radiative decay of polyatomic molecules.

Intramolecular coupling, intramolecular relaxation and no observable radiative interference effects are expected in the following cases:

(a) Intramolecular radiationless decomposition. In the well understood cases of predissociation and autoionization we encounter a conventional 
relaxation phenomenon. Line broadening is observed and the branching ratio for fluorescence is lower than unity. Obviously, the reduction of emission is a much more sensitive criterion for radiationless decomposition than line broadening.

(b) The statistical limit. In large molecules the dense quasi-continuum can be considered for all practical purposes as an effective decay channel. Line broadening and intramolecular relaxation effects are exhibited in this limit.

Intramolecular coupling will be exhibited while no intramolecular relaxation and no radiative interference effects will be observed in the following cases:

(c) Accidental degeneracy of two levels corresponding to different electronic terms in a diatomic molecule. A small molecule may exhibit the effects of strong vibronic perturbations between pairs of accidentally degenerate levels. These perturbations considerably exceed the radiative width. A complex spectrum results which is sensitive to external fields; however no radiative interference effects will be exhibited. A typical example involves ${ }^{2} \Sigma-{ }^{2} \mathrm{II}$ mixing in the $\mathrm{CN}$ molecule ${ }^{58}$.

(d) Sparse intermediate case. The density of vibronic states in the $\left\{\phi_{l}\right\}$ manifold is rather small $(\rho \sim 1 \mathrm{~cm})$; however the coupling matrix elements are large. The situation corresponds to the coarse strongly coupled distribution discussed in section IV. These small molecules will exhibit long radiative lifetime ${ }^{35}$.

Finally we have to consider the circumstances whereupon radiative interference effects will (or may) be observed:

(e) The resonance limit. A pair of levels which split by intrinsic or external perturbations and which are spaced within their radiative widths will exhibit quantum beats in the radiative decay.

(f) The dense intermediate case. A small electronic energy gap in a large molecule (e.g. the second excited singlet state of napthalene and pyrene which are separated by $3000 \mathrm{~cm}^{-1}$ from the first singlet). In this case one has to consider separately the weakly and strongly coupled levels in the vibronic manifold $\left\{\phi_{l}\right\}$. Under these circumstances the width of the zero order state is shared between several closely spaced resonances. Several interesting effects can now be encountered for the radiative decay resulting from intersystem crossing in the isolated molecule which corresponds to this situation. Emission will take place from the highly excited vibronic component of the lowest singlet to high vibronic components of the ground state.

The following effects may be observable ${ }^{33}$ :

(1) 'Lengthening' of the radiative lifetime of some strongly coupled components.

(2) A possible observation of quantum beats due to interference between a small number of closely spaced levels.

(3) Non-exponential decay due to 'smearing out' of the interference effects, when the number of the strongly coupled levels is too large (but insufficient for the validity of the statistical limit).

(4) Effects of external fields on the level mixing and the decay characteristics.

(5) All these phenomena will be erased when the molecule is embedded in a dense medium in view of external vibrational relaxation effects. 


\section{RADIATIONLESS TRANSITIONS}

In summary, we have presented in Table 5 the experimental phenomena related to intramolecular coupling and intramolecular relaxation while Table 6 presents some of the characteristic features of the radiative decay of small, medium sized and large molecules.

Table 5. Experimental phenomena related to intramolecular coupling and relaxation in molecules

\begin{tabular}{ccccc}
\hline Classification & $\begin{array}{c}\text { Radiationless } \\
\text { decomposition } \\
\text { predissociation } \\
\text { autoionization } \\
\text { (a) }\end{array}$ & $\begin{array}{c}\text { Sparse level } \\
\text { distribution }\end{array}$ & $\begin{array}{c}\text { Dense } \\
\text { intermediate } \\
\text { case }\end{array}$ & $\begin{array}{c}\text { Statistical } \\
\text { limit }\end{array}$ \\
\hline
\end{tabular}

\begin{tabular}{lcccc}
\hline System & $\begin{array}{l}\text { Small and } \\
\text { large molecules }\end{array}$ & $\begin{array}{l}\text { (a) }{ }^{2} \pi{ }^{2} \Sigma \\
\text { in } \mathrm{CN}\end{array}$ & $\begin{array}{l}\text { Small gap in } \\
\text { large molecules }\end{array}$ & $\begin{array}{l}\text { Large gap in } \\
\text { large molecules } \\
\text { case (c) in solution }\end{array}$ \\
$\begin{array}{c}\text { Experimental } \\
\text { methods }\end{array}$ & $(1), 2,(3), 6$ & $1,2,3,4,5$ & $1,2,3,4(5)$ & $1,2,3,4,6$ \\
$\begin{array}{c}\text { Intramolecular } \\
\text { interstate } \\
\text { coupling }\end{array}$ & + & + & + & + \\
$\begin{array}{c}\text { Radiative } \\
\text { interference }\end{array}$ & - & - & $(+?)$ & - \\
$\begin{array}{c}\text { Intramolecular } \\
\text { relaxation }\end{array}$ & + & - & $(+?)$ & + \\
\hline
\end{tabular}

Experimental methods: (1) Decay times

(2) Line shapes or intensity distribution in absorption

(3) Fluorescence yields

(4) Fluorescence spectra

(5) External yields

(6) Population of final state

\section{THE NON-RADIATIVE DECAY PROBABILITY AND THE ENERGY GAP LAW IN THE STATISTICAL LIMIT}

The theory outlined in the preceding sections provides a unified formal description of electronic relaxation processes in large molecules. However, this formalism will be viewed with suspicion by the experimentalist as it does not provide predictions of the non-radiative decay probability. Furthermore, we have been concerned up to this point with electronic relaxation processes and have paid no attention to photochemical rearrangement reactions. Recently, a general theory of non-radiative processes was considered in the statistical limit by Lin and Bersohn ${ }^{26}$ and by Englman and Jortner ${ }^{59 a}$, and by Freed and Jortner ${ }^{59 b}$; this is based on the following assumptions:

(a) A two electronic-level system was considered, consisting of a small number of levels $\phi_{s 1}, \phi_{s 2} \ldots$ etc. (the dynamic part) and the dissipative channel $\left\{\phi_{l j}\right\}$. The second index lables the vibrational states. The general form of the BO functions is

$$
\phi_{\alpha \beta}\left(r, Q^{(\alpha)}\right)=\Theta_{\alpha}\left(r, Q^{(\alpha)}\right) \chi_{\alpha \beta}\left(Q^{(\alpha)}\right)
$$

where $\boldsymbol{r}$ represents the electronic coordinates, $Q^{(\alpha)}$ labels the nuclear normal coordinates in the electronic state $\alpha$, while $\Theta_{\alpha}$, and $\chi_{\alpha \beta}$ represent electronic and vibrational wave functions. 
Table 6. General features of the decay of molecular levels.

\begin{tabular}{|c|c|c|c|c|}
\hline $\begin{array}{l}\text { Physical } \\
\text { Property }\end{array}$ & $\begin{array}{l}\text { Resonance } \\
\text { Limit }\end{array}$ & $\begin{array}{c}\text { Sparse } \\
\text { Intermediate Case }\end{array}$ & $\begin{array}{c}\text { Dense } \\
\text { Intermediate Case }\end{array}$ & $\begin{array}{l}\text { Statistical } \\
\text { Limit }\end{array}$ \\
\hline $\begin{array}{l}\text { Number of states } \\
N=(\Delta / 2 \varepsilon)=\pi V^{2} \rho^{2}\end{array}$ & - & $N>1$ & $N \sim 1$ & $N \gg 1$ \\
\hline $\begin{array}{l}\text { Level separation } \\
\text { relative to radiation } \\
\text { width }\end{array}$ & $\varepsilon \sim \Gamma_{s}$ & $\rho^{-1} \gg \Gamma_{s}$ & $\rho^{-1} \sim \Gamma_{s}$ & $\rho^{-1} \ll \Gamma_{s}$ \\
\hline \multirow[t]{2}{*}{ Line shape } & $\begin{array}{l}\text { Natural radiative } \\
\text { + conventional } \\
\text { broadening }\end{array}$ & $\begin{array}{l}\text { Intensity distribution, } \\
\text { well separated lines }\end{array}$ & Intermediate structure & Lorentzian line shape \\
\hline & & & & $\Delta=\pi V^{2} \rho$ \\
\hline $\begin{array}{l}\text { relative to } \\
\text { recurrence time }\end{array}$ & $t \sim h / \varepsilon$ & $t \gg \hbar \rho$ & $t \sim h \rho$ & $t \ll h \rho$ \\
\hline Mode of decay & Beat spectrum & $\begin{array}{l}\text { Sum of (slowly varying) } \\
\text { exponentials }\end{array}$ & $\begin{array}{l}\text { Oscillatory (beats) } \\
(?)\end{array}$ & Exponential \\
\hline $\begin{array}{l}\text { Mean radiative } \\
\text { decay time }\end{array}$ & - & $\tau_{e} \sim\left(N \hbar / \Gamma_{s}\right)$ & $\tau_{e} \sim \hbar / \Gamma_{s}(?)$ & $\tau_{e}=\left(\hbar / \Delta+\Gamma_{s}\right)$ \\
\hline $\begin{array}{l}\text { Experimental } \\
\text { fluorescence } \\
\text { quantum yield }\end{array}$ & $Y=1$ & $Y=1$ & $Y=1$ & $Y=\left(\Gamma_{s} / \Gamma_{s}+\Delta\right)$ \\
\hline $\begin{array}{l}\text { Features of } \\
\text { relaxation }\end{array}$ & External & External & External & $\begin{array}{l}\text { Intramolecular } \\
\tau_{N R}=\hbar / \Delta\end{array}$ \\
\hline Examples & $\begin{array}{l}\text { Level crossing } \\
\text { Level anticrossing }\end{array}$ & $\mathrm{SO}_{2}, \mathrm{NO}_{2}, \mathrm{CS}_{2}$ & $?$ & $\begin{array}{l}\text { Anthracene } \\
\text { Tetracene }\end{array}$ \\
\hline
\end{tabular}


(b) Interference effects between the compound states constructed from each of the components of the dynamic part are disregarded. Hence, the non-radiative decay probability is displayed in the form:

$$
\tau_{n r}^{-1}=\frac{2 \pi}{\hbar} \sum_{i} \sum_{j}\left|V_{s i, l j}\right|^{2} p(s i) \delta\left(E_{s i}-E_{l j}\right)
$$

where $p(s i)$ is the population of the initial state $|s i\rangle$ and the coupling matrix elements are

$$
V_{s i, l j}=\left\langle\phi_{s i}\left|H_{v}\right| \phi_{l j}\right\rangle
$$

(c) It was assumed that the normal modes and their frequencies are the same in the two electronic states except for displacements in the origin.

Let the normal coordinates be denoted by $Q_{j}(j=1 \ldots N)$ with the effective masses $M_{j}$ and frequencies $\omega_{j}$. The equilibrium configuration of the electronic state $s$ is characterized by the configuration $Q_{j}^{(s)}(j=1 \ldots N)$. Let $\Delta Q_{j}^{(0)}=$ $Q_{j}^{0(l)}-Q_{j}^{0(s)}$ correspond to the displacement of the $Q_{j}$ normal coordinate in the equilibrium configuration of the electronic state $l$ relative to the electronic state $s$. It will be also useful to define a set of dimensionless coordinates and displacements:

$$
\begin{gathered}
q_{j}=\left(M_{j} \omega_{j} / \hbar\right)^{\frac{1}{2}}\left(Q_{j}-Q_{j}^{0(s)}\right) \\
\Delta_{j}=\left(M_{j} \omega_{j} / \hbar\right)^{\frac{1}{2}} \Delta Q_{j}^{(0)}
\end{gathered}
$$

(d) The molecular vibrations are harmonic and anharmonicity effects were disregarded. The adiabatic potentials $W_{s}$ and $W_{1}$ for the two electronic states are then given in the form:

$$
\begin{gathered}
W_{s}=\frac{1}{2} \sum_{j} \hbar \omega_{j} q_{j}^{2} \\
W_{l}=\frac{1}{2} \sum_{j} \hbar \omega_{j}\left(q_{j}-\Delta_{j}\right)^{2}-\Delta E=W_{s}-\sum_{j} \gamma_{j} q_{j}-\Delta E+E_{M}
\end{gathered}
$$

The most important energy parameter introduced at this point is the energy gap between the lowest vibronic components of the two electronic states:

$$
\Delta E=E_{\mathrm{s} 0}-E_{l 0}
$$

$\gamma_{j}=\hbar \omega_{j} \Delta_{j}$ corresponds to the linear coupling term for the $j$ th mode. The energy term

$$
E_{M}=\frac{1}{2} \sum_{j} \hbar \omega_{j} \Delta_{j}^{2}
$$

represents the molecular nuclear relaxation energy, or rather half the Stokes shift, due to the reduced displacement $\Delta_{j}$.

These assumptions seem to be rather pedestrian and were in fact used before. In spite of considerable activity in the field conventional computa- 
tional methods as employed before cannot be expected to yield reliable information for a large molecule which is characterized by a large number, $N$, of vibrational degrees of freedom. In view of the complexity of the problem encountered in the calculation of $\tau_{n r}^{-1}$ in the statistical limit, conventional 'quantum chemistry' type methods seem to be inadequate. General problems related to the calculation of expressions of the form of equation (IX.2) were encountered in solid state physics. Such a task was considered by Lamb ${ }^{60}$ and by others ${ }^{61,62}$ for the Mössbauer effect. The nuclear recoil problem for the displacements in the momentum space requires the same treatment as a harmonic lattice. Indeed, analogous problems were encountered in the theory of line shapes and zero phonon lines in the absorption spectra of impurity centres in solids ${ }^{63,64}$. Finally, similar methods were introduced ${ }^{65,66}$ for the study of radiationless transitions (e.g. thermal ionization) in solids. Englman, Freed and Jortner attempted to consider the problem of radiationless transitions in a large molecule from the point of view of multiphonon processes. Indeed, for a large molecule when $N \gg 1$ it seems a logical step to transfer the problem from the field of molecular physics to the realm of solid state physics and to consider the problem of 'phonons in large molecules'.

Two physically interesting cases have to be considered at this point.

(a) When the molecule is inserted into an inert medium which acts as a heat bath, thermal equilibrium among the $s i$ levels can be assumed. Provided that the vibrational relaxation (and excitation) rates considerably exceed the non-radiative decay times we can set:

$$
p(s i)=\exp \left(-\beta E_{s i}\right) / \sum_{i} \exp \left(-\beta E_{s i}\right)
$$

where $\beta=\left(k_{B} T\right)^{-1}$, so that when thermal equilibrium prevails, one has

$$
\tau_{n r}^{-1}=2 \pi / \hbar \sum_{i} \sum_{j} \exp \left(-\beta E_{s i}\right)\left|V_{s i, l j}\right|^{2} \delta\left(E_{s i}-E_{l j}\right) / \sum_{i} \exp \left(-\beta E_{s i}\right)
$$

(b) For the case of an isolated molecule (in vacuum or, even better, in outer space) we can consider a coherent excitation of a single vibronic state $\phi_{s i}$, whereupon $p(s i)=\delta_{i, i^{\prime}}$. When this zero order vibronic level $s i^{\prime}$ corresponds to $s i$ the transition probability is given by (IX.11) in the zero temperature limit (or rather for $\beta \rightarrow \infty$ ).

Now, the approximate expressions for the non-radiative transition derived above are completely analogous to the formal expressions for the line shape in optical absorption in solids. Indeed, in this approximation the non-radiative process can be formally regarded as a (symmetry forbidden) optical emission process in the limit of zero energy. Equation (IX.11) can be handled by the application of the generating function method. The main advantage of this technique is that it handles the generalized density of states function of the form (IX.11) (e.g. the density of states weighted by an arbitrary operator) without the necessity of factoring out these expressions into products of matrix elements and the vibronic density of states.

The non-radiative transition probability can be recast in the form of a Fourier transform:

$$
\tau_{n r}^{-1}=C^{2} / \hbar^{2} \exp (-G) \int_{-\infty}^{\infty} \mathrm{d} t \exp \left[(-i \Delta E t / \hbar)+G_{+}(t)+G_{-}(t)\right]
$$


where $C$ is the non-adiabatic coupling matrix element ${ }^{58,59}$ and the functions $G_{ \pm}(t)$ are given by

and

$$
\begin{aligned}
G_{+}(t) & =\frac{1}{2} \sum_{j} \Delta_{j}^{2}\left(\bar{n}_{j}+1\right) \exp \left(i \omega_{j} t\right) \\
G_{-}(t) & =\frac{1}{2} \sum_{j} \Delta_{j}^{2} \bar{n}_{j} \exp \left(-i \omega_{j} t\right) \\
\bar{n}_{j} & =\left[\exp \left(\beta \hbar \omega_{j}\right)-1\right]^{-1}
\end{aligned}
$$

$\bar{n}_{j}$ is the number of excited vibrations with frequency $\omega_{j}$ at thermal equilibrium. The dimensionless quantity $G$ is defined in the form

$$
G=G_{+}(0)+G_{-}(0)=\frac{1}{2} \sum_{j} \Delta_{j}^{2}\left(2 \bar{n}_{j}+1\right)
$$

which corresponds to the change in the number of vibrational quanta in the radiationless transition. This quantity is referred to as the coupling strength. The general result derived herein can be recast in a more transparent form for certain limiting cases, which are determined by the magnitude of the coupling strength $G$ (equation (IX.15)). In order to obtain an approximate estimate for the coupling strength we make use of equation (IX.9) and write the approximate relation

$$
G \approx E_{M} / \hbar\langle\omega\rangle \operatorname{coth}(\beta \hbar\langle\omega\rangle / 2)
$$

where $\langle\omega\rangle=N^{-1} \sum_{j} \omega_{j}$ is the mean vibrational frequency. The low and high temperature limits are :

$$
\begin{aligned}
& G=E_{M} / \hbar\langle\omega\rangle ; \quad \beta \hbar\langle\omega\rangle \gg 1 \\
& G=2 E_{M} / \beta(\hbar\langle\omega\rangle)^{2} ; \quad \beta \hbar\langle\omega\rangle \ll 1
\end{aligned}
$$

The various coupling limits can be defined as follows :

(a) On the strong coupling limit $G \geqslant 1$ or alternatively,

$$
G \gg \hbar\langle\omega\rangle \operatorname{coth}(\beta \hbar\langle\omega\rangle / 2) \text {. }
$$

At low temperatures the strong coupling limit will be encountered whereupon $E_{M}$ exceeds the mean vibrational frequency so that the relative displacement of the potential energy surfaces are large, and the Stokes shift will considerably exceed the vibrational frequency. Under these circumstances it is possible for the energy surfaces of the two electronic states to cross or to intersect in the vicinity of the minimum of the upper surface. Such a situation was examined thirty years ago by Teller ${ }^{67}$.

(b) The weak coupling limit is encountered when $G \ll 1$ or (at low temperatures) $E_{M} \ll \hbar\langle\omega\rangle$. Thus the relative displacement for each normal mode is relatively small.

In the strong coupling limit the general expression can be reduced to a closed form:

$$
\tau_{n r}=\frac{C^{2}(2 \pi)^{\frac{3}{2}} \exp \left[-\left(\Delta E-E_{M}\right)^{2} / 4 E_{M} k_{B} T^{*}\right]}{\hbar\left(2 E_{M} k_{B} T^{*}\right)^{\frac{1}{2}}}
$$


where the effective temperature is defined in the form:

$$
k_{B} T^{*}=\frac{1}{2} \hbar\langle\omega\rangle \operatorname{coth}(\beta \hbar\langle\omega\rangle / 2)
$$

The Gaussian dependence on the energy parameter $\left(\Delta E-E_{M}\right)$ in the strong coupling limit is of course analogous to the Gaussian line shape (near the band maximum) devoid of phonon structure for impurity centres. However, equation (IX.20) contains some further interesting information. Inspection of equations (IX.6) and (IX.7) reveals that the potential surfaces $W_{s}$ and $W_{1}$ intersect on the hypercurve (actually on the (N-1)-dimensional surface). The intersection point of minimum energy $E_{A}$ measured from the energy origin $E_{s 0}=0$ is

$$
E_{A}=\left(\Delta E-E_{M}\right)^{2} / 4 E_{M}
$$

The following result is finally obtained in the strong coupling limit

$$
\tau_{n r}^{-1}=\frac{C^{2}(2 \pi)^{\frac{1}{2}}}{\hbar\left(E_{M} k_{B} T^{*}\right)^{\frac{1}{2}}} \exp \left(-E_{A} / k_{B} T^{*}\right)
$$

This equation has the general appearance of a conventional rate equation where the energy $E_{A}$ plays the role of the activation energy as might have been guessed by the intelligent chemist on intuitive grounds. It is important to stress that the concept of the 'activated complex' does not enter in any way into this treatment. The rate equation results from quantum mechanical transition between greatly displayed potential surfaces.

In the weak coupling limit the integral (IX.12) can be evaluated by the method of steepest descent. The low temperature result is to the lowest $\operatorname{order}^{58,59}$

$$
\begin{aligned}
\tau_{n r}^{-1} & =\frac{C^{2}(2 \pi)^{\frac{1}{2}}}{\hbar\left(\hbar \omega_{M} \Delta E\right)^{\frac{1}{2}}} \exp \left(-\gamma \Delta E / \hbar \omega_{M}\right) \\
\gamma & =\log \left(\frac{\Delta E}{\mathrm{~d} e_{M}}\right)-1
\end{aligned}
$$

where $\omega_{M}$ and $\Delta_{M}$ are the frequencies and the reduced displacement of the modes of maximum frequency (e.g. the $\mathrm{C}-\mathrm{H}$ or $\mathrm{C}-\mathrm{D}$ modes) and

$$
\mathrm{d} l_{M}=\frac{1}{2} \sum_{M=1}^{\mathrm{d}} \hbar \omega_{M} \Delta_{M}^{2} .
$$

This equation immediately exhibits :

(a) The energy gap law.

(b) The isotope effect in the weak coupling limit.

The main accomplishments of this treatment can be summarized as follows :

(a) From the general structure of the theoretical formulae we can ascertain the relative displacement of the two potential energy surfaces expressed in terms of the coupling parameter $G$ determines whether the molecular system corresponds to the strong or to the weak coupling limit. This classification provides a link between the Teller picture ${ }^{67}$ and the conventional tunnelling model for radiationless transitions. In the statistical limit, as it is wellestablished, the weak (and possibly sometimes the intermediate) coupling 


\section{RADIATIONLESS TRANSITIONS}

scheme is appropriate for the description of a large number of radiationless transitions (e.g. electronic relaxation) in aromatic molecules.

(b) In the strong coupling limit the transition probability is determined by the mean molecular frequency, provided, of course, that the $\Delta_{i}$ values for a substantial number of different frequencies are non-vanishing. On the other hand, in the weak coupling case the non-radiative transition probability is dominated by the highest molecular frequency $\omega_{M}$

(c) In the strong coupling limit the transition probability is determined by the energy $E_{A}$ corresponding to the point of minimum intersection energy located above the origin of the higher electronic state. In the weak coupling limit the transition probability is essentially determined by the energy gap $\Delta E$.

(d) A proper theoretical interpretation of the "energy gap law' for radiationless transitions in the weak coupling limit is provided. This general energy-difference dependent behaviour is characteristic of many molecular relaxation processes, such as vibrational relaxation.

(e) Following the considerations presented in (b), some features of the intramolecular isotope effect on radiationless transitions can be elucidated. A pronounced isotope effect can be encountered only in the weak coupling limit.

(f) Medium effects resulting from coupling to an inert medium are now elucidated $^{59}$.

(g) From the chemist's point of view the different features of the isotope effects, the energy gap law and the temperature dependence encountered in the weak and strong coupling limits can be summarized as follows: the weak coupling limit corresponds to a tunnelling mechanism between zero order vibronic levels which correspond to different electronic configurations, while in the strong coupling limit we encounter the situation in which adiabatic potential surfaces cross or intersect. It is gratifying that both limits results as particular cases of the same general formalism.

Although conventional radiationless transitions in large aromatic molecules correspond to the weak coupling situation, the strong coupling limit is of considerable physical interest for the interpretation of many reactions encountered in the field of organic photochemistry.

\section{REFERENCES}

${ }^{1}$ For a review, see G. Herzberg. Spectra of Diatomic Molecules (Van Nostrand and Co., 1954), Vol. II, p. 406.

${ }^{2}$ For reviews, see:

(a) M. Kasha. Discussions Faraday Soc. 9, 14 (1950).

(b) M. Kasha. Radiation Res. Suppl. 2, 243 (1960).

(c) P. Seybold and M. Gouterman. Chem. Rev. 65, 413 (1965).

(d) B. R. Henry and M. Kasha. Ann. Rev. Phys. Chem. 19, 161 (1968).

(e) J. Jortner, S. A. Rice and R. M. Hochstrasser. Advan. Photochem. In press (1971).

3 A. Wiedeman. Ann. Phys. 34, 446 (1888).

${ }^{4}$ A. Jablanski. Nature 131, 839 (1933).

5 G. N. Lewis and M. Kasha. J. Am. Chem. Soc. 66, 2100 (1944); 67, 994 (1945).

6 G. W. Robinson and R. P. Frosch. J. Chem. Phys. 37, 1962 (1962); 38, 1187 (1963).

(a) C. A. Hutchinson Jr and B. W. Magum. J. Chem. Phys. 32, 1261 (1960).

(b) M. S. de Groot and J. H. Van der Waals. Mol. Phys. 4, 189 (1961).

${ }^{8}$ M. Gouterman. J. Chem. Phys. 36, 2846 (1962). 


\section{JOSHUA JORTNER}

9 R. J. Watts and S. J. Strickler. J. Chem. Phys. 44, 2423 (1966).

10 R. Williams and G. J. Goldsmith. J. Chem. Phys. 39, 2008 (1963).

11 W. R. Ware and P. T. Cunningham. J. Chem. Phys. 44, 4364 (1966).

12 A. B. Zahlan, S. Z. Weisz, R. C. Jarmagin and M. Silver. J. Chem. Phys. 42, 4244 (1965),

13 (a) G. B. Kistiakowski and C. S. Parmeter. J. Chem. Phy's. 42, 2942 (1965).

(b) E. M. Anderson and G. B. Kistiakowsky. J. Chem. Phys. 48, 4787 (1968).

(c) A. E. Douglas and W. Mathews. J. Chem. Phys. 48, 4788 (1968).

14 G. W. Robinson. J. Chem. Phys. 47, 1967 (1967).

15 M. Bixon and J. Jortner. J. Chem. Phys. 48, 715 (1968).

16 U. Fano. Phys. Rev. 124, 1866 (1961).

17 O. K. Rice. Phys. Rev. 33, 478 (1929).

18 C. A. Coulson and K. Zalewski. Proc. Roy. Soc. (London) A268, 437 (1962).

19 R. A. Harris. J. Chem. Phys. 39, 978 (1963).

20 G. Herzberg. Electronic Spectra of Polyatomic Molecules (Van Nostrand \& Co., Princeton, N.J., 1966).

21 J. Franck and H. Sponer. Gottingen Nachr. (1928), p. 241.

22 R. Kubo. Phys. Rev. 86, 929 (1952).

23 E. F. McCoy and I. G. Ross. Australian J. Chem. 15, 573 (1962).

${ }^{24}$ G. R. Hunt, E. F. McCoy and I. G. Ross. Australian J. Chem. 15, 591 (1962).

25 J. P. Byrne, E. F. McCoy and I. G. Ross. Australian J. Chem. 18, 1589 (1965).

${ }^{26}$ S. H. Lin. J. Chem. Phys. 44, 3759 (1966).

27 P. C. Haarhoff. Mol. Phys. 7, 101 (1963).

28 J. Jortner and R. S. Berry. J. Chem. Phys. 48, 2757 (1968).

29 D. Chock, J. Jortner and S. A. Rice. J. Chem. Phys. 49, 610 (1968).

${ }^{30}$ M. Bixon, J. Jortner and Y. Dothan. Mol.Phys. 17, 109 (1969).

${ }^{31}$ M. Bixon and J. Jortner. J. Chem. Phys. 50, 4061 (1969).

${ }^{32}$ M. Bixon and J. Jortner. J. Chem. Phys. 50, 3284 (1969).

${ }^{33}$ K. F. Freed and J. Jortner. J. Chem. Phys. 50, 2916 (1968).

${ }^{34}$ H. C. Longuet-Higgins. Advan. Spectr. 2, 429 (1961).

${ }^{35}$ H. Feshbach. A. K. Kerman and R. H. Lemmer. Ann. Phys. (N.Y.) 41, 230 (1967).

${ }^{36}$ A. E. Douglas. J. Chem. Phys. 45, 1007 (1966); A. E. Douglas and K. P. Huben. Can. J. Phys. 43, 74 (1965).

37 J. P. Byrne and I. G. Ross. Can. J. Chem. 43, 3253 (1965).

${ }^{38}$ G. R. Hunt and I. G. Ross. J. Mol. Spect. 9, 50 (1962).

39 P. G. Wilkinson. Can. J. Phys. 34, 596 (1956).

40 D. S. McClure. J. Chem. Phys. 22, 1968 (1954).

41 J. G. Angus, B. J. Christ and G. C. Morris. Australian J. Chem. In press.

42 J. Jortner and G. C. Morris. J. Chem. Phys. 51, 3689 (1969).

${ }^{43}$ K. Freed. J. Chem. Phys. 51, (1970).

${ }^{44}$ W. Hanle. Z. Physik 30, 93 (1924).

45 G. Breit. Rev. Mod. Phys. 5, 91 (1933).

${ }^{46}$ P. A. Franken. Phys. Rev. 121, 508 (1961).

47 M. E. Rose and R. L. Corovillano. Phys. Rev. 122, 1185 (1961).

48 T. G. Eck. Physica 33, 157 (1967).

49 H. Wieder and T. G. Eck. Phys. Rev. 153, 103 (1967).

50 R. L. Kelly. Phys. Rev. 147, 376 (1966).

51 J. N. Dod, R. D. Kaul and D. M. Warrington. Proc. Phys. Soc. (London) 84, 176 (1964).

52 E. B. Alexandrov. Optics and Spectroscopy 17, 957 (1967).

53 J. N. Dodd, W. J. Sandle and D. Zisserman. Proc. Phys. Soc. (London) 92, 797 (1967).

${ }^{54}$ W. Weisskopf and W. Wigner. Z. Physik 63, 54 (1930).

55 K. E. Lassila. Phys. Rev. 135A, 1218 (1964).

${ }^{56}$ R. P. Feynman, R. B. Leighton and M. Sands. The Feynman Lectures on Physics (Addison Wesley, 1965), Vol. 3, Chapter 11-5.

57 J. S. Bell and J. Steinberger. Proceedings of the Oxford International Conference on Elementary Particles (1965), p. 195.

${ }^{58}$ H. E. Radford and H. P. Broida. J. Chem. Phys. 38, 644 (1963); Phys. Rev. 128, 231 (1963).

5.) (a) R. Englman and J. Jortner. Mol. Phys. 18, 145 (1970).

(b) K. Freed and J. Jortner. J. Chem. Phys. 52, 6272 (1970).

60 W. E. Lamb. Phys. Rev. 55, 190 (1938). 


\section{RADIATIONLESS TRANSITIONS}

61 W. H. Visscher. Ann. Phys. 9, 194 (1960).

62 K. S. Singwi and A. Sjölander. Phys. Rev. 120, 1093 (1960).

${ }^{63}$ K. Huang and A. Rhys. Proc. Phys. Soc. (London) A204, 413 (1951).

${ }^{64}$ M. Lax. J. Chem. Phys. 20, 1752 (1952).

65 R. C. O'Rourke. Phys. Rev. 91, 265 (1953).

66 R. Kubo. Phys. Rev. 86, 929 (1952).

${ }^{67}$ E. Teller. J. Phys. Chem. 41, 109 (1937). 